\title{
Correction: the buccohypophyseal canal is an ancestral vertebrate trait maintained by modulation in sonic hedgehog signaling
}

\author{
Roman H Khonsari ${ }^{1,2,15^{*}+}$, Maisa Seppala ${ }^{1,3^{*}+}$, Alan Pradel ${ }^{4}$, Hugo Dutel ${ }^{5,6}$, Gaël Clément ${ }^{5}$, Oleg Lebedev ${ }^{7}$, \\ Sarah Ghafoor ${ }^{1}$, Michaela Rothova ${ }^{1,8}$, Abigael Tucker ${ }^{1}$, John G Maisey ${ }^{4}$, Chen-Ming Fan ${ }^{9}$, Atsushi Ohazama ${ }^{1}$, \\ Paul Tafforeau ${ }^{10}$, Brunella Franco ${ }^{11}$, Jill Helms ${ }^{12}$, Courtney J Haycraft ${ }^{13}$, Albert David ${ }^{14}$, Philippe Janvier $^{5}$, \\ Martyn T Cobourne $e^{1,3^{*}+}$ and Paul T Sharpe ${ }^{1 * \dagger}$
}

\section{Correction}

The authors noted that the figure legend for Figure 1 (parts (a) and (b)) needs correcting [1].

The Figure 1 legend should read:

Figure 1 Rathke's pouch is located at a triple boundary. a) Whole-mount LacZ staining of an R26R-Sox17-Cre E10.5 mouse embryo showing the anterior limit of the endoderm (red arrowhead) and the posterior border of Rathke's pouch (blue arrowhead); endobuccal view of the oral roof. (b) Posterior limit (arrowhead) of the neural crest-derived mesenchyme in the mid-sagittal plane at E12.5, corresponding to the location of the closing buccohypophyseal canal, in an R26R-Wnt1-Cre mouse embryo. (c) Anterior limit of the endoderm in the mid-sagittal plane at E10.5 (arrowhead), LacZ staining, R26RSox17- Cre mouse embryo. (d) Anterior end of the notochord (arrowhead), eosin staining, E10.5, wild-type mouse embryo ant, anterior; post; posterior; RP, Rathke's pouch.

Please also note the contact address for Philippe Janvier should be: 5.CNRS-UMR 7207, Muséum national d'Histoire naturelle-UPMC, Paris, France.

\footnotetext{
* Correspondence: roman.khonsari@kcl.ac.uk; maisa.seppala@kcl.ac.uk; martyn. cobourne@kcl.ac.uk; paul.sharpe@kcl.ac.uk

${ }^{\dagger}$ Equal contributors

'Department of Craniofacial Development and Stem Cell Research, Comprehensive, Biomedical Research Center, Dental Institute, King's College London, London, UK

${ }^{3}$ Department of Orthodontics, Dental Institute, King's College London,

Guy's Hospital, London, UK

Full list of author information is available at the end of the article
}

\begin{abstract}
Author details
'Department of Craniofacial Development and Stem Cell Research, Comprehensive Biomedical Research Center, Dental Institute, King's College London, London, UK. ²Service de Chirurgie Maxillo-Faciale, Centre Hospitalier Universitaire Hôtel-Dieu, Nantes, France. ${ }^{3}$ Department of Orthodontics, Dental Institute, King's College London, Guy's Hospital, London, UK. ${ }^{4}$ American Museum of Natural History, New York, USA. ${ }^{5}$ CNRS-UMR 7207, Muséum national d'histoire naturelle-UPMC, Paris, France. ${ }^{6}$ CNRS-UMR 7179, Muséum national d'histoire naturelle, Paris, France. ${ }^{7}$ Paleontological Institute of Russian Academy of Science, Moscow, Russian Federation. ${ }^{8}$ Institute of Experimental Medicine, Academy of Sciences of the Czech Republic, Prague, Czech Republic. ${ }^{9}$ Department of Embryology, Carnegie Institution of Washington, Baltimore, USA. ${ }^{10}$ European Synchrotron Radiation Facility, Grenoble, France. ${ }^{11}$ Department of Pediatrics, Università degli Studi di Napoli Federico II, Naples, Italy. ${ }^{12}$ Department of Surgery, Stanford University, Palo Alto, USA. ${ }^{13}$ College of Dental Medicine, Craniofacial Biology, Medical University of South Carolina, Carolina, USA. ${ }^{14}$ Service de Génétique clinique, Centre Hospitalier Universitaire Hôtel-Dieu, Nantes, France. ${ }^{15}$ Department of Craniofacial Development and Stem Cell Biology Dental Institute, King's College London, Guy's Hospital, London SE1 9RT, UK.
\end{abstract}

Received: 13 June 2013 Accepted: 13 June 2013

Published: 21 June 2013

\section{Reference}

1. Khonsari RH, Seppala M, Pradel A, Dutel H, Clément G, Lebedev O, Ghafoor S, Rothova M, Tucker A, Maisey JG, Fan CM, Kawasaki M, Ohazama A, Tafforeau P, Franco B, Helms J, Haycraft CJ, David A, Janvier P, Cobourne MT, Sharpe PT: The buccohypophyseal canal is an ancestral vertebrate trait maintained by modulation in sonic hedgehog signaling. BMC Biology 2013, 11:27.

doi:10.1186/1741-7007-11-70

Cite this article as: Khonsari et al:: Correction: the buccohypophyseal canal is an ancestral vertebrate trait maintained by modulation in sonic hedgehog signaling. BMC Biology 2013 11:70.

\section{C) Biomed Central}

(c) 2013 Khonsari et al.; licensee BioMed Central Ltd. This is an Open Access article distributed under the terms of the Creative Commons Attribution License (http://creativecommons.org/licenses/by/2.0), which permits unrestricted use, distribution, and reproduction in any medium, provided the original work is properly cited. 